\title{
JÓVENES CONECTADOS: UNA APROXIMACIÓN AL OCIO DIGITAL DE LOS JÓVENES ESPAÑOLES.
}

\author{
Connected young: An aproach to digital leisure of spanish youth.
}

\author{
Autores: VIÑALS BLANCO, Ana; ABAD GALZACORTA, Marina y \\ AGUILAR GUTIÉRREZ, Eduardo
}

\author{
Investigadora Predoctoral Gobierno Vasco - Universidad de Deusto-España-ana.vinals@deusto.es \\ Profesora Doctora - Universidad de Deusto - España-marina.abad@deusto.es \\ Profesor Licenciado - Universidad de Deusto-España-eaguilar@deusto.es
}

\section{Resumen}

El desarrollo de las Tecnologías de la Información y de la Comunicación (TIC) e Internet ha transformado la manera en la que los jóvenes experimentan y disfrutan de su ocio, dando pie al impulso de un ocio digital propio de la sociedad red. Con base a una revisión de los principales estudios realizados hasta el momento en materia de ocio, jóvenes y TIC, este artículo analiza las prácticas de ocio de los jóvenes españoles. Jóvenes nacidos en plena era digital, rodeados de pantallas y que adquieren unas características diferenciadas de sus progenitores y docentes. Jóvenes multitarea, conectados, prosumidores, sociales y móviles que han adoptado las herramientas digitales como algo fundamental en sus actividades de ocio relacionadas principalmente con la socialización y el entretenimiento. En este sentido, se puede concluir que el ocio juvenil se caracteriza por ser por un lado, un ocio digital social en el que predomina el uso de las redes sociales virtuales, por otro lado, un ocio digital móvil posibilitado por la conectividad sin límites que ofrecen los smartphone y, por último, un ocio digital lúdico ligado al uso de los videojuegos. Sin embargo, y desde el punto de vista del ocio humanista, este artículo aborda el gap existente entre esa aproximación y la visión comercial de los informes de medición del ocio.

\section{Palabras clave}

Jóvenes, ocio digital, redes sociales virtuales, smart-phones, videojuegos

\begin{abstract}
The development of Information and Communication Technologies (ICT) and the Internet has transformed the way in which young people experience and enjoy their leisure, giving rise to the impulse of digital leisure proper to network society.

On the basis of a literature review of the main studies carried out so far in leisure, youth and ICT, this article analyzes the leisure practices of Spanish young people. Young born in digital era, surrounded by screens and who acquire distinguishing characteristics of their parents and teachers. Multitasking, online, prosumers, social and mobile young people who embrace digital tools as fundamental in their leisure activities primarily related to socialization and

In this sense, it can be concluded that the young people leisure is characterized by, on the one hand, a social digital leisure in the predominant use of virtual social networks, on the other hand, a mobile digital leisure possible by connectivity limits provided by the smartphone and, finally, a ludic digital leisure linked to the use of video games. However, from the viewpoint of humanistic leisure, this article addresses the existing gap between this approach and the commercial vision of the measurement reports of leisure.
\end{abstract}

\section{Key words}

Young people, digital leisure, social networks, smart-phones, videogames 


\section{Introducción}

En base a una conceptualización del ocio como una "experiencia gratuita, necesaria y enriquecedora de la experiencia humana” (Cuenca, 2000:15), este artículo trata de describir las características del ocio propio de la realidad digital ante la que se encuentran los jóvenes españoles. Jóvenes que, a pesar de la diferencia entre las edades englobadas, se hallan en una etapa de transición a la vida adulta muy significativa en su desarrollo como personas y que han nacido en plena revolución digital. Es innegable que la irrupción de las Tecnologías de la Información y de la Comunicación (en adelante TIC) e Internet en el día a día ha supuesto cambios significativos en prácticamente todas las actividades diarias, y el ámbito del ocio no ha podido quedar al margen. Para la generación de jóvenes que se está desarrollando de forma paralela a esta realidad digital, y a los que se denomina como nativos digitales (Prensky, 2001), Millennials (Howe y Strauss, 2000) o Generación A (Coupland, 2010) entre otros, ha significado un cambio en la forma de vivir el ocio. De ahí, que también haya suscitado el interés del ámbito académico en entender qué factores influyen en la conducta hacia el uso de la tecnología y en qué medida. Sin embargo, todavía existen grandes lagunas de conocimiento y metodológicas en su aplicación en entornos de ocio (Abad, 2009), más aun desde una concepción del ocio, como ocio experiencial.

\section{Objetivos}

En este contexto la finalidad de este artículo es dar respuesta a tres objetivos entrelazados: 1) explorar cómo es el ocio de los jóvenes españoles; 2) analizar de qué manera han influido los avances tecnológicos en sus prácticas de ocio, y 3) extraer qué características en relación con esa dimensión digital les define. En última instancia lo que se pretende es establecer una radiografía que nos permita analizar las prácticas de ocio de los jóvenes nacidos en plena era digital, sin perder de vista la necesaria reflexión evaluación sobre las fuentes de datos vigentes y las variables utilizadas en los diferentes informes desde un punto de vista del ocio humanista. Por lo tanto, se trata de reflexionar sobre su idoneidad a la hora de dar respuesta a preguntas claves como: ¿qué hacen los jóvenes españoles en su tiempo libre?, ¿para qué y cómo utilizan la tecnología y los recursos digitales?; ¿están las TIC e Internet redefiniendo los ocios de la población juvenil? y en definitiva, ¿qué rasgos definen a los jóvenes nacidos en la era digital?

\section{Metodología}

Para dar respuesta a estos interrogantes se ha optado por realizar una revisión de los informes y estudios (fuentes primarias y secundarias) hasta el momento realizados en la temática del ocio juvenil ligado al uso de herramientas digitales. Un análisis que, además de permitirnos dar respuesta a los objetivos planteados anteriormente y hacer una aproximación al ocio digital de los jóvenes, también ha supuesto la base para ponderar la posibilidad de analizar esas prácticas de ocio desde la perspectiva más conceptual del ocio experiencial. Esto permite una reflexión novedosa y original del fenómeno del ocio digital.

El artículo se estructura en cuatro grandes apartados. En primer lugar, se expone la manera de entender el ocio de la que se parte, esto es, el ocio como expe- riencia. Asimismo, se analiza el modo en el que la tecnología ha influido en el disfrute del ocio y en la proliferación de 'nuevos' ocios ligados a la red (en línea). En segundo lugar, se expone una perspectiva general del ocio juvenil, ahondando en el tiempo libre del disponible y en las actividades de ocio más practicadas por los jóvenes. En tercer lugar, y en base a los datos anteriormente expuestos, se destacan tres tendencias de ocio predominantes entre los jóvenes en las que el papel de herramientas y soportes digitales ocupa un papel central: el ocio digital social (redes sociales virtuales), el ocio digital móvil (smartphone) y el ocio digital lúdico (videojuegos). En cuarto lugar, y a modo de conclusión, se aporta una reflexión en torno a la nueva forma de interpretar el ocio juvenil, esto es, un ocio digital transmedia 
y multiplataforma como forma de consumo/prosumo (Toffler, 1970). Un ocio que, de alguna manera, engloba las tres tendencias de ocio anteriormente expuestas. Un tipo de ocio que deja entre- ver las características que definen a una generación de jóvenes multitarea, conectados, sociales, digitales, móviles, transmedia y multisoporte.

\section{4. ¿Qué entendemos por ocio?}

La relevancia actual del fenómeno del ocio se manifiesta tanto en el desarrollo económico y social como en el valor que damos al tiempo libre disponible. De unos años a esta parte, la influencia de las TIC e Internet en el ocio ha desembocado en un cambio sustancial en la forma en la que disfrutamos de nuestro tiempo libre. La sociedad en red ha traído consigo una variación en las percepciones del tiempo y el espacio y, en la actualidad, las experiencias de ocio se asientan bajo un espacio de flujos, de constantes cambios y un tiempo que se ha vuelto atemporal (Castells, 2001).

\subsection{El ocio como experiencia: una forma de entender el ocio}

Como resultado de las continuas transformaciones sociales sucedidas a lo largo de los últimos años, el ocio, como valor en sí mismo, se ha convertido en un importante pilar de desarrollo humano en el siglo XXI (Cuenca, 2010). Pero su importancia es aún mayor ya que "el ocio también posibilita la experimentación de valores vitales muy positivos para el desarrollo de la vida humana como la libertad, satisfacción, gratuidad, identidad, superación, formación y justicia” (Cuenca, 2011:44). Analizar cuáles son las actividades de ocio más practicadas por los jóvenes objeto de este estudio nos aporta información valiosa acerca de cómo son realmente, por la influencia que tiene el ocio en la construcción de la personalidad individual. Asimismo, profundizar en sus prácticas de ocio nos puede ayudar a distinguir qué características concretas les definen.

Por otro lado, el significado atribuido al ocio ha variado considerablemente a lo largo del tiempo. Así por ejemplo, el ocio ha sido concebido como un tiempo para el descanso, para la diversión, para la desconexión de las rutinas diarias, como tiempo libre dedicado a hacer algún tipo de actividad, como una fuente de salud y prevención de enfermedades físicas y psíquicas, como un signo de calidad de vida tanto a nivel personal como social o como una fuente importante de valor económico, entre otras. En otras palabras, no es lo mismo concebir el ocio como la práctica de una mera actividad, como tiempo libre disponible o como ociosidad y por ello, resulta esencial definir desde un principio de qué concepción de ocio parte este estudio.

El concepto trabajado se enmarca en una forma particular de entender el ocio propio del Instituto de Estudios de Ocio de la Universidad de Deusto que promueve una perspectiva multidisciplinar e integral del ocio. En dicho Instituto se investiga el ocio experiencial (Cuenca, Aguilar y Ortega, 2010:4455), fundamentado en "la vivencia de experiencias personales complejas (direccionales y multidimensionales), centradas en actuaciones queridas (libres y satisfactorias), autotélicas (con un fin en sí mismas) y personales (con implicaciones individuales y sociales)" (Cuenca, 2006:14). Dicho de otro modo, un ocio positivo, satisfactorio, elegido libremente por la persona y cuya práctica se encuentra separada de finalidades útiles. Una visión particular de entender el ocio que se define de manera concreta en la Carta Internacional para la Educación en Ocio adoptada por el Consejo de la Asociación Mundial del Ocio y la Recreación en 1993.

Por ello, nos fundamentamos en una noción de ocio entendida como experiencia humana libre, gozosa y con fin en sí misma. Experiencias de ocio que siendo vivencias humanas personales y subjetivas adoptan una serie de rasgos comunes en las que el marco de referencia es la propia persona. Toda experiencia de ocio tiene un predominio emocional y el ocio es una vivencia que se encuadra en el mundo de las emociones y que se practica porque 'me gusta'. Un argumento poco racional, pero un signo de afirmación personal respecto a los demás que posibilita la expresión de los propios deseos y gustos. 
En este sentido, tomamos como punto de partida un ocio que no se justifica en el deber y se realiza básicamente porque agrada $\mathrm{y}$, por eso, tiene un sentido. Aún más, el ocio no sólo es un valor en sí mismo, sino que además es un valor subordinado a otros más amplios como la felicidad o la autorrealización personal. Es por ello, que las experiencias de ocio son de carácter procesual, es decir, transcurren en una temporalidad tridimensional al fijar su realidad en el presente, pero enriquecerse en la medida que incorporan significativamente el previo (preparación) y el post (recuerdos). Por lo tanto, el ocio se experimenta en distintos niveles de intensidad, en función de si se tratan de actividades que simplemente nos gustan practicar o bien son prácticas de ocio que suponen una inmersión receptiva y contemplativa capaz de proporcionarnos una experiencia intensa e inolvidable. Esta distinción se sustenta en la formación previa que se tenga en lo que habilidades de ocio se refiere, a saber, en la capacidad que se tenga de disfrutar del ocio (Cuenca, 2009).

Del mismo modo, el concepto de ocio es un área de análisis complejo e interdisciplinar que difícilmente puede ser abarcable desde el conocimiento de una sola de las disciplinas académicas tradicionales. En función de la persona, sus circunstancias y orientación, el ocio aporta diferentes beneficios y consecuencias positivas relacionadas con el alivio de conductas de insatisfacción, el estrés, el aburrimiento, la falta de actividad física o de creatividad. Cuestiones que, desafortunadamente, están muy presentes en el contexto actual. De cualquier modo, queda evidenciado que este modo de entender el ocio, ligado a la capacidad de experimentación del mismo en el sentido más subjetivo, no encaja con la tendencia social y la manera de concebirlo existente, ya que en la realidad se tiende a asociar el ocio a parámetros meramente objetivos, de consumo y a relacionarlo con la práctica de diferentes actividades, en tiempos y espacios concretos ya preestablecidos. Sin embargo, desde la perspectiva de análisis que aquí se plantea, lo importante radica en la vivencia de experiencias de ocio memorables, satisfactorias y transformadoras.

En este sentido, se pone de manifiesto una de las primeras limitaciones metodológicas del estudio, ya que cuando se acude a las fuentes de datos relativas a jóvenes y ocio como el informe "Jóvenes y ocio digital" (UOC, 2009), "La Sociedad de la Información en España” (Fundación Telefónica,2012) o "Cifras y jóvenes" (INJUVE, 2011) se aprecia que el concepto de ocio en el que desarrollan las preguntas y respuestas no queda bien definido y más importante aún, la conceptualización del ocio que se contempla se sitúa muy lejos del marco conceptual que defiende este estudio (véase Cuadro ${ }^{\circ} 1$ ).

\section{Cuadro 1: Diferentes variables de medición del uso de Internet vinculado al ocio.}

\begin{tabular}{|c|c|c|}
\hline $\begin{array}{l}\text { Jóvenes y ocio digital } \\
\text { (UOC, 2009) } \\
\text { Uyer de intemé }\end{array}$ & $\begin{array}{c}\text { Informe SIE } \\
\text { (Telefónica, 2012) } \\
\text { Contesidor digitrales coasuo } \\
\text { altemutivas de oclo }\end{array}$ & $\begin{array}{l}\text { Cifras y jóvenes } \\
\text { (INJuvE, 2011) } \\
\text { ¿Para qué utilizass } \\
\text { principolmente lateme? }\end{array}$ \\
\hline $\begin{array}{l}\text { - Baszel rote } \\
\text { - Buscar información } \\
\text { sobre trahajos o } \\
\text { deberes del colegio o } \\
\text { del instituto } \\
\text { - Bajar música, peliculas } \\
\text { o videojuegos; } \\
\text { lnformarse sobre temas } \\
\text { que interesen como } \\
\text { videojuegos, viajes, } \\
\text { moda,.. } \\
\text { - Para relacigazrse con la } \\
\text { gente } \\
\text { - Ver o escuchar pro-. } \\
\text { gramas de TV, racio, } \\
\text { videos musicales } \\
\text { - Distribuir fotos, videos } \\
\text { que tú hagas o tus } \\
\text { opiniones } \\
\text { - Buscar información } \\
\text { sobre sexualidad, } \\
\text { drogas o el aspecto } \\
\text { fisico. }\end{array}$ & 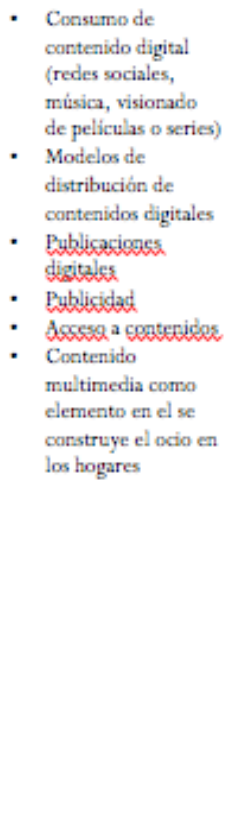 & 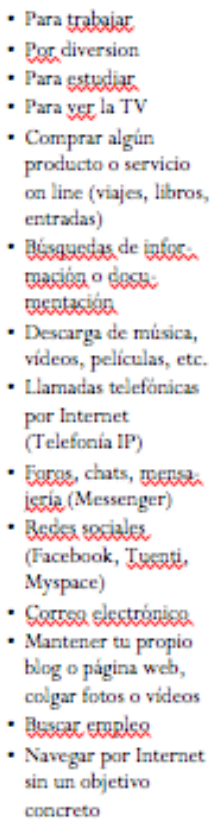 \\
\hline
\end{tabular}

Fuente: Elaboración propia

Si bien, para conocer lo que el ocio significa para la persona que lo experimenta resulta necesario, primeramente, identificar las prácticas de ocio que realiza según parámetros objetivos. A partir de ahí, y si la persona las percibe como tal, entonces preguntar por las actividades que más les gustan y las que más le gustaría realizar. En definitiva, en este artículo se defiende un enfoque de ocio diferenciado del consumo, y se apuesta por valorar las experiencias que se disfrutan, lo que supone entender el ocio como una experiencia humana, básica y vital, para lo 
cual es necesario entender cómo es el ocio en la actualidad.

\subsection{El ocio en una sociedad digital y conectada}

El desarrollo de la cultura digital no sólo ha propiciado nuevas prácticas de ocio, sino que también ha transformado la propia forma de practicarlas. Factores externos tales como la inmediatez, la cultura del entretenimiento, de la imagen, de la extimidad (frente a intimidad) y del consumo en general (Sibilia, 2008) han influido en el ámbito del ocio, situándolo en el contexto de una sociedad postmoderna fundamentada en el "aquí y ahora". Más aún, el desarrollo imparable de todo un entramado de herramientas tecnológicas cada vez más accesibles y fáciles de usar y la democratización del uso de Internet de banda ancha ha dado pie a la generación de un ocio que responde al modelo social imperante. Un tipo de ocio que, como plantea San Salvador del Valle (2009), ha pasado a concebirse como un fast-ocio que implica una desaforada búsqueda del aprovechamiento al límite del tiempo percibido como bien escaso. Un ocio enmarcado en una "sociedad líquida" (Bauman, 1999) cuyos principales rasgos son el cambio constante, la transitoriedad, la fragilidad de los vínculos humanos y la incertidumbre.

El impulso de una sociedad en red en perpetuo movimiento, donde lo que realmente importa es estar conectados, ha desembocado en que vivir en una permanente conexión con los demás se convierta en un valor significativo para las personas. Este hecho ha evidenciado el temor social que existe a la soledad (Turkle, 2012), el aburrimiento y a la desconexión.

La red forma ya parte de nuestras vidas y el número de internautas continúa en aumento, en especial, entre los jóvenes de 16 a 24 años para quienes Internet se ha instaurado como esencial en sus vidas. Además, el espacio virtual o ciberespacio alberga "nuevos" ocios "simultáneos" y "atemporales" que han pasado a formar parte de la cultura de la virtualidad real, esto es, una cultura en la que "no hay separación entre la "realidad" y la "representación simbólica" (Castells, 2007: 405).

Es lógico que el ocio propio de una sociedad conectada sea un ocio apoyado en el uso de herramientas tecnológicas que, en gran parte, debido al desarrollo imparable de los smartphones, ha derivado en un disfrute del ocio desligado de contextos concretos, adaptándolo a los diferentes y variados tiempos sociales de la vida. De hecho, el ocio ha pasado a concebirse en "pequeñas píldoras de fruición, brevedades que pueden disfrutarse en los microespacios que dejan las actividades laborales o en los fragmentos de dedicación ociosa que el usuario se adjudica durante los desplazamientos o en su tiempo libre en el hogar" (Igarza, 2009:43). Esto es, "burbujas de ocio" que para este autor forman parte de un tiempo de ocio intersticial, interactivo, más selectivo, menos duradero y basado en formatos micro. Donde el entretenimiento, el placer, la distensión, el esparcimiento y la diversión es lo que prima.

Sin embargo, y pese a los rasgos propios de un contexto social líquido, la realidad nos muestra a través de numerosos ejemplos que la red de redes ofrece una serie de oportunidades que pueden transformar estas "burbujas de ocio" en un ocio con sentido y beneficioso. El espacio virtual brinda la ventaja de tornarse en un espacio idóneo para la participación, la colaboración, la creación de conocimiento colectivo, el aprendizaje entre pares, etc. Espacios de ocio digital en red que lejos de instaurarse como meras herramientas y espacios virtuales de comunicación, socialización, entretenimiento y diversión, pueden constituirse al mismo tiempo como entornos de aprendizaje, de desarrollo humano y de mejora de la calidad de vida individual y social.

Por lo tanto, ¿qué entendemos por ocio digital?, ¿nuevos ocios digitales ligados al uso de la red? ¿ocios tradicionales que se han tecnologizado? ¿simplemente es una nueva forma de denominar al ocio propio de la era digital? En definitiva, ¿podemos hablar de ocio digital?, ¿supone el componente virtual un plus en la vivencia de ocio?

\subsection{Hacia "nuevas" tipologías de ocio}

Las actividades de ocio ligadas al uso de la tecnología e Internet comúnmente se engloban bajo diferentes denominaciones tales como ocio online (vs offline), ocio virtual (vs presencial) u ocio digital (vs analógico), por lo tanto, casi todas lo son por 
oposición. Del mismo modo, la palabra "nuevo" es un término absolutamente relativo y vinculado al punto de vista del lector, por lo que en este caso se utiliza para diferenciar los usos objeto de análisis frente a los no vinculados a la irrupción tecnológica. Por ello, este epígrafe se centra en describir estos "nuevos ocios en red".

Desde un enfoque amplio del término entendemos por ocio digital a todas las oportunidades de ocio que se encuentran ligadas a las posibilidades que ofrece el uso de las tecnologías digitales. Esto es, el ocio que se disfruta y experimenta a través del uso del ordenador, Internet, las consolas de juegos, los teléfonos móviles y los diversos dispositivos digitales emergentes producto del desarrollo constante e innovador de la industria tecnológica: los Ipad, las tabletas, los MP3, los e-books, y un largo etcétera. Diferentes herramientas y soportes tecnológicos que, en última instancia, han modificado la experiencia de ocio al ofrecerle un añadido en cuanto a interactividad, conectividad, hipertextualidad, comodidad, anonimato, ubicuidad etc. Ahora bien, ¿qué características posee este ocio ligado a la red?, ¿es posible definirlo correctamente empleando como referencia los parámetros tradicionales de análisis del ocio o se trata de un tipo de ocio que asume cualidades propias?

Tradicionalmente, el ocio ha sido analizado básicamente tomando en consideración tres aspectos básicos: el tiempo y el espacio, la actividad y la experiencia (Katz et al., 2000; Kelly, 1996 citados en Nimrod y Adoni, 2012). Unos parámetros que hasta el momento nos han ayudado a comprender mejor las características de las prácticas de ocio. Pero, ¿son aplicables al contexto digital actual? (Nimrod y Adoni, 2012).

En primer lugar, las variables de "tiempo", "espacio", "frecuencia" y "duración" han sido los parámetros que han marcado la diferencia en las actividades de ocio llevadas a cabo hasta el momento. Así pues, discernir entre el lugar de las prácticas de ocio, el tiempo social en el que se desarrollan, el número de veces que se practica determinada acción y el tiempo invertido en la misma vislumbra claramente una distinción en los hábitos de ocio. Este criterio continúa manteniéndose en el análisis del ocio en red, más aún cuando en la actualidad el tiempo de ocio es ubicuo y no se entiende únicamente como el tiempo libre que se emplea fuera del trabajo, en fines de semana o periodos vacacionales, sino como el tiempo que también se dispersa entre la propia jornada laboral y los quehaceres diarios. Las TIC nos permiten estar en varios sitios a la vez e identificar el tiempo y espacio de las prácticas de ocio es un requisito fundamental también en el análisis de los "nuevos ocio en línea".

En segundo lugar, las variables de "lo social", "la participación" y "la formación" han sido también importantes aspectos que han aportado información rica al análisis del ocio. Discernir entre prácticas de ocio individuales, sociales y en comunidad, entre un ocio activo o pasivo (tanto física como mentalmente) y conocer el grado de formación o no en habilidades y competencias que posibilitan disfrutar del ocio han sido puntos que claramente diferenciaban unas prácticas de ocio de otras. Pautas que igualmente sirven para marcar la diferencia en las actividades de ocio en línea. En este sentido, en la actualidad son muy comunes los estudios realizados en torno a la temática de las brechas digitales (participación) o la alfabetización mediática (formación). Aspectos ligados al uso de las TIC e Internet.

Por último, también es posible analizar la "experiencia de ocio" del e-ocio desde la misma perspectiva utilizada hasta el momento. Ya que el nivel de implicación (ocio serio y ocio casual), la motivación (intrínseca o extrínseca) y los beneficios percibidos (psicológicos, fisiológicos, psicofisiológicos, sociales, económicos y medioambientales) tanto en actividades tradicionales de ocio, como en actividades practicadas en espacios virtuales continúan siendo aspectos que determinan el valor, valioso o no, del ocio.

En síntesis, "los tres aspectos fundamentales del ocio - el tiempo, la acción y la experiencia - al igual que muchos de sus aspectos relacionados, son comunes a ambos tipos de ocio, el tradicional y el online" (Nimrod y Adoni, 2012:40) ${ }^{1}$.

Sin embargo, las actividades de ocio practicadas en espacios virtuales se diferencian de las actividades de

\footnotetext{
1"'... the three core aspects of leisure - time, action and experience - as well as many of their related dimensions, are common to both traditional and E-leisure" (Nimrod y Adoni, 2012: 40).
} 
ocio offline en que poseen unos rasgos específicos propios del contexto en que se practican y que las enriquecen. Al llevarse a cabo en el ciberespacio, las características inherentes de este, es decir, la sincronicidad, la interactividad, el anonimato y la participación en la realidad virtual dotan de unos rasgos específicos a estas actividades de e-ocio (Nimrod y Adoni, 2012).

De esta manera, las actividades de ocio en línea pueden ser tanto sincrónicas como asincrónicas, esto es, se pueden llevar cabo en tiempo real y de manera instantánea (chat) o bien establecerse de forma no simultánea (correo electrónico). Asimismo, son actividades de por sí interactivas (Van Dijk, 1999) debido a que se desarrollan en medios de comunicación asincrónicos y no lineales, rasgos clave para que se establezca interactividad propia de la red. Asimismo, este tipo de prácticas de ocio en red posibilitan "intercambios recíprocos de comunicación que implican el uso de algún tipo de medio de comunicación o tecnología de la información y de la comunicación” (Bucy, 2008:375 en Nimrod y Adoni, 2012:42). Ofrecen la posibilidad de ser anónimas, cualidad exclusiva del ocio mediado por pantallas. El anonimato es un rasgo que generalmente es considerado de manera negativa, debido a que los usuarios pueden neutralizar sus rasgos personales off line, mostrándose como personas diferentes, es decir, lo que viene a denominarse como el deviant leisure (Rojek y Urry, 2000). Si bien, al mismo tiempo, este anonimato puede ayudar a que ciertas personas se muestren tal y como son al margen de estereotipos externos.

Por último, las autoras exponen que el ocio en línea es el único tipo de ocio que ofrece la posibilidad de participación en la realidad virtual. Internet cada vez es más social y otorga poder al usuario, quien tiene en su mano el disfrute de participar en la colaboración, modificación, creación e intercambio de información. Este rasgo es valorado de manera positiva por los usuarios, quienes resaltan la opción de estar físicamente en un lugar, pero virtualmente en otro completamente diferente. El ocio virtual ofrece una continuidad de experiencias y es por ello que se integra en la vida cotidiana como una parte más de esta. Con todo, ¿que consideramos por ocio digital?, ¿actividades de ocio que han sido atravesadas por el paradigma digital o espacios digitales que han sufrido la influencia de la cultura tradicional? o ¡tal vez el ocio digital en sí mismo no existe y es más correcto hablar de ocios posibilitados por herramientas digitales?

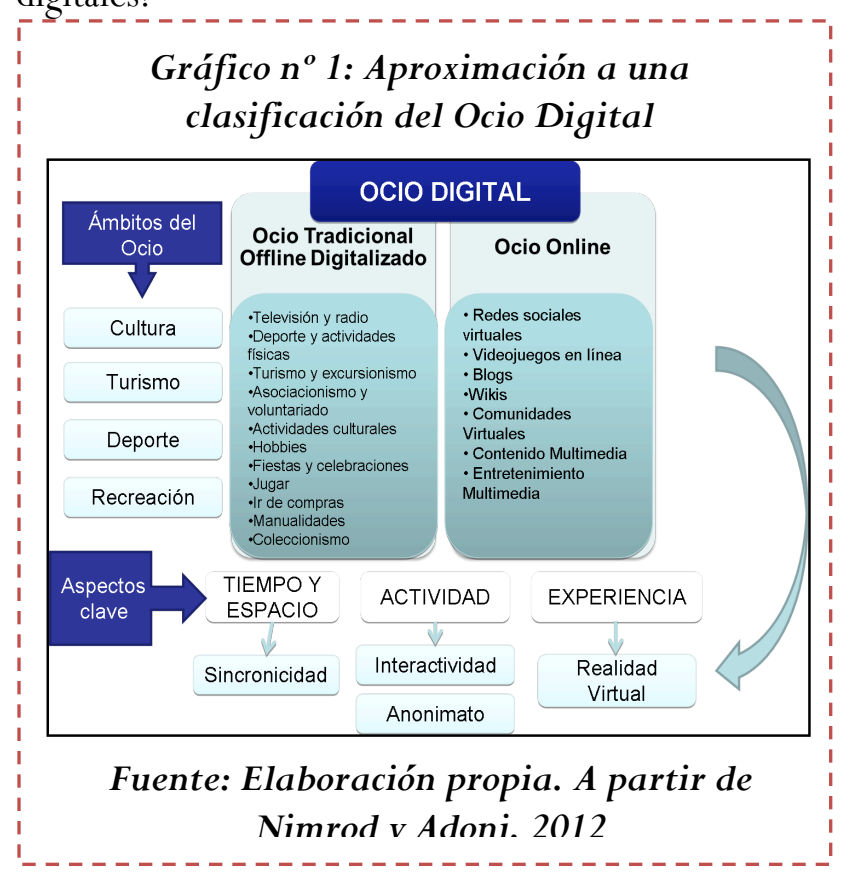

Son numerosas las interpretaciones posibles al respecto, no obstante, en este trabajo se recurre a la denominación de "ocio digital" como concepto general que engloba por un lado, las actividades de ocio tradicionales offline que se han tecnologizado y, por otro lado, las actividades practicadas en contextos virtuales (Véase Gráfico.n ${ }^{\circ} 1$ ). Por actividades de ocio tradicionales off line tecnologizadas nos estaríamos refiriendo a las tradicionales actividades de ocio que en la era digital se han transformado (lectura - ebooks), y en cuanto a las actividades de ocio online hacemos referencia al conjunto de actividades de ocio que posibilita la red (redes sociales virtuales, blogs, wikis, contenidos multimedia, la participación en foros, la colaboración en comunidades virtuales etc.).

Si bien, a la hora de distinguir entre espacios de ocios físicos y virtuales, simplemente pretendemos hacer referencia a la confección de nuevos espacios y contextos de ocio que han posibilitado nuevas oportunidades de disfrutar del tiempo libre. Es decir:

“interpretamos el ocio digital en Internet no como un estar conectado (ocio online) frente a no conectado (ocio offline), sino como seguir conectado al mundo social digitalmente construido, no como un ocio virtual frente a un ocio real, 
sino como un nuevo espacio donde continuar las relaciones sociales" (García, E.; López, J. y Samper, A., 2012:397).

El e-ocio no es por tanto una sustitución del ocio offline, simplemente se trata de un tipo de ocio emergente en espacios virtuales, que dada a las características que este aporta (sincronicidad, interactividad, anonimato y participación en la realidad virtual) está transformando las experiencias de ocio. En definitiva, por ocio digital entendemos un continuo de experiencias, tanto experiencias virtuales que se integran en la vida diaria, como experiencias offline que traspasan al ciberespacio. Se trata de un ocio que aporta los mismos beneficios que el ocio tradicional, que "aporta desconexión y escape de las tensiones de la vida diaria y (...) puede ayudarnos en

la mejora de la salud y la confección de nuestra identidad personal" (Bryce, 2001: 8).

Dicho de otro modo, consideramos aquí el ocio digital de manera integral, un ocio offline que se ha visto influenciado por el desarrollo de las TIC y un ocio virtual que se desarrolla en espacios digitales. Siendo conscientes de que, en última instancia, lo fundamental es que estos ocios emergentes, ligados al uso de Internet, se configuren como ocios positivos en los que las personas disfruten y se desarrollen como personas. Ocios digitales conectados, activos, creativos y solidarios que sean referentes de identidad, superación y justicia, alejados del mero entretenimiento y pasar el rato.

\section{El ocio de los jóvenes españoles}

Intentar caracterizar a los jóvenes y su ocio nos lleva ineludiblemente a reflexionar no sólo entorno al contexto actual que les rodea (era digital, cambiante, inestable, precaria, compleja etc.) sino también sobre la cultura juvenil y sus características concretas. Castells et al. (2007) afirman que la cultura juvenil es:

"el sistema específico de valores y creencias que conforman el comportamiento de un grupo de edad en concreto y que muestra una serie de características distintivas en relación con otros grupos de edad de la sociedad. Dicha cultura debe situarse en el contexto de una estructura social dada. Es decir, no nos referimos aquí al análisis histórico sobre el significado de juventud, sino a la especificidad cultural de la juventud en la estructura social que caracteriza nuestro tiempo: la sociedad en red" (Castells et al., 2007:205).

De nuevo se pone de manifiesto otra limitación metodológica de este acercamiento y es las diferencias existentes en las principales fuentes de datos relativas a jóvenes y ocio ya que mientras INJUVE identifica los jóvenes entre 15 y 19 años, el informe de "Jóvenes y ocio digital"(UOC, 2009) se centra en la franja entre 12 y 18 años (diferenciando entre 12-15 y 16-18 años). Del mismo modo, no siempre se tratan de informe periódicos, por lo que las informes no siempre son coetáneos ni actuales.

\subsection{Tiempo libre disponible y penetra- ción de las TIC en la vida}

Según la Cuarta Encuesta Europea de Valores (2010) en su aplicación a España, en los últimos años, el valor asignado al ocio se ha revalorizado frente al del trabajo, convirtiéndose así el ocio en un importante pilar de desarrollo humano. Según el Informe Juventud en España (INJUVE, 2010), los jóvenes varones de entre 15 y 19 años disponen de una media de 34,5 horas de tiempo libre a la semana y las mujeres 33,9 horas. Lo que supone que los jóvenes disponen de una media de 5 horas diarias de tiempo libre. Ahora bien, ¿esta disponibilidad temporal se correlaciona con la disponibilidad subjetiva? En base a los datos extraídos del último Informe Juventud en España (2012) mientras el 29\% de los y las jóvenes afirman tener más de 39 horas libres disponibles a la semana, el $23 \%$ reconoce menos de 16 . Lo que confirma que pese a tener una gran cantidad de horas disponibles, los jóvenes perciben que éstas son muchas menos.

Al mismo tiempo, la penetración de las TIC en la vida ha propiciado un importante cambio cultural. El 94\% de las personas dispone de telefonía móvil, el $84 \%$ tiene telefonía fija, el $67 \%$ Internet y un $24 \%$ cuenta con el servicio de televisión de pago (ONTSI, 2012). El smartphone o teléfono inteligente ha experimentado un incremento interanual de 8,4 puntos porcentuales 
alcanzando una penetración del 41,5\% entre los individuos de 15 años y más años, y el 66,9\% de los hogares dispone de acceso a Internet. El número de individuos con algún móvil activo aumenta de manera constante y el chat (whatsapp, mensajería instantánea,...) ha experimentado un importante incremento de uso. El 41\% de los usuarios de móvil disponen de esta funcionalidad y el $71 \%$ la utiliza a diario.

Las tecnologías se han instaurado como referentes fundamentales en el tiempo social de ocio, y correlativamente en un espacio de consumo juvenil (INJUVE, 2012). Por lo que se puede afirmar que la penetración de la tecnología además de en la vida cotidiana, también ha pasado a ocupar una parte importante del tiempo libre. La mayoría de las personas jóvenes considera que Internet es muy o bastante útil para la vida cotidiana, pero mucho más el teléfono móvil. De ahí que se considere al teléfono móvil como el principal exponente del uso tecnológico de las personas jóvenes siendo su uso prácticamente universal. Asimismo, mientras una mayoría de los jóvenes cree que estas tecnologías aíslan, vuelven perezosas a las personas, hacen perder mucho tiempo y reducen la eficiencia personal, al mismo tiempo opinan que frente a cualquier otro tipo dispositivo tecnológico el teléfono móvil representa la libertad, independencia y la posibilidad de permanente contacto y disponibilidad (INJUVE, 2012).

Entre tanto, el estudio muestra cómo el uso del ordenador entre los jóvenes también ha aumentado, siendo paralelo el incremento en el uso de Internet. La conexión es cada vez mayor desde dispositivos portátiles y ha crecido exponencialmente desde los teléfonos móviles. El informe concluye que el acceso a la red no es sólo cada vez más numeroso, sino también más frecuente: el $93 \%$ de los y las jóvenes dice acceder a diario y el $87 \%$ varias veces al día. El tiempo dedicado a Internet resta del tiempo dedicado a otras actividades, pero fundamentalmente al tiempo dedicado a no hacer nada (INJUVE, 2012).

\subsection{Prácticas de ocio de los jóvenes es- pañoles}

Según los datos comentados en los párrafos anteriores, los jóvenes de entre 15 y 19 años disponen de 5 horas de media de tiempo libre diarias y la tecnología forma una parte considerable de este tiempo. Ahora bien, ¿que realizan los jóvenes en este tiempo libre?, ¿qué papel ocupa la tecnología?

Se observa que las actividades de tiempo libre mayoritarias y casi universales de los jóvenes se ligan con el aspecto relacional y tecnológico (INJUVE, 2012). De esta manera, salir a reunirse con los amigos, escuchar música, ver la televisión y usar el ordenador son las cuatro actividades más practicadas por los jóvenes españoles de entre 15 y 19 años. Además, "se constata una alta acomodación entre las actividades que más gustan y las que más se realizan, siendo además esta práctica frustrada tanto menos cuanto más mayoritaria es la actividad" (INJUVE, 2012:313).

Asimismo, si reflexionamos sobre la manera en que el desarrollo de las TIC e Internet ha influido en las prácticas de ocio, se puede afirmar que todas las actividades han adquirido de alguna $u$ otra manera un importante input tecnológico o digital. Así por ejemplo, los jóvenes se socializan y comunican a través de la red, la música se escucha a través del mismo teléfono móvil, dispositivos MP3 o en línea, el modo de ver la televisión y de jugar se ha tornado digital etc. Inmersos en una cultura digital, las prácticas de ocio offline tradicionales o bien se han tecnologizado, o bien se han trasladado al espacio virtual. Estos "nuevos" ocios se siguen contemplando y analizando en base a los parámetros de tiempo, espacio, actividad y experiencia, pero en la era digital estas prácticas se han vuelto ubicuas, sincrónicas, interactivas, anónimas y participativas en red. Aspectos que claramente han transformado la manera de experimentar del ocio, provocando la aparición de un nuevo proceso: la digitalización de la experiencia de ocio.

Los últimos estudios en torno al ocio juvenil destacan una serie de prácticas que se están convirtiendo en auténticos fenómenos sociales, constituyendo una tendencia especialmente significativa en este colectivo. 


\section{Tendencias de ocio y perfil de los jóvenes de la era digital}

Tal y como se viene afirmando la cada vez mayor influencia de las TIC en la vida cotidiana se refleja de igual forma en el ocio, especialmente en lo referente a las actividades relacionadas directamente con los procesos de socialización y entretenimiento. Esta nueva situación ha desencadenado en auténticas transformaciones y "nuevos ocios" que han ido adquiriendo especial incidencia en el colectivo juvenil dando lugar a nuevas tendencias de ocio que destacan por su gran penetración entre los jóvenes y que podríamos denominar como ocio transmedia. Aunque en algunos casos son fenómenos simultáneos, ya que por ejemplo lo social no está reñido con lo móvil, sino todo lo contrario, entre las macrotendencias tecnológicas y por su importancia de afectación, se aborda esta realidad desde tres puntos de vista: el uso de las redes sociales virtuales, el fenómeno de los videojuegos y por último,lo que ha supuesto la movilidad, entendida como ubicuidad, para el entretenimiento incluyendo los dos fenómenos anteriores.

\subsection{Ocio Digital Social: redes sociales virtuales}

El uso de la redes sociales virtuales (en adelante RSV) se ha convertido en uno de los hábitos sociales más practicados entre los jóvenes. La democratización de Internet y la accesibilidad móvil, facilitada por los smartphone, la ha instaurado como una práctica cotidiana ubicua (Iab Spain, 2013). A pesar de que las RSV son simplemente herramientas tecnológicas que conectan a personas a través de diferentes medios digitales, su uso se ha convertido en una auténtica revolución social y de masas entre los jóvenes, principalmente en lo que al ámbito de la comunicación, la socialización, la participación y el entretenimiento se refiere.

El uso de las redes sociales se ha generalizado entre las personas jóvenes en poco más de tres años. En 2009 las utilizaban el 60\% de los jóvenes, y en 2011 su uso alcanzaba el 90\% (INJUVE, 2012). Asimismo, entre los jóvenes de 15 a 29 años que son usuarios de Internet destacan la búsqueda de información o documentación (82,0\%), el estar en redes sociales $(79,6 \%)$ y usar el correo electrónico
$(76,3 \%)$ como sus tres principales usos de Internet. Por lo que podemos decir que informarse, comunicarse y entretenerse son las tres motivaciones de uso de la red (INJUVE, 2011). El uso de este tipo de redes es mayoritariamente diario $y$, con frecuencia, son medios sociales destinados a la "hipercomunicación": comunicar, informar, construir, mantener la red de contactos, compartir, participar, colaborar, cotillear etc.

Facebook es la red social más utilizada (Iab Spain, 2013) con un total de 1.060 millones de usuarios registrados, seguida de Youtube, Twitter y Tuenti. Estas redes se ajustan a las características y edades de los usuarios, de ahí que el uso de unas redes, en un momento, se vaya sustituyendo por el uso de otras de manera constante. Tuenti se concibe como el entorno virtual propio de la jóvenes-adolescentes (14 -16 años) y Facebook es la red social por excelencia de los jóvenes-adultos (16-24 años).

Las RSV se han constituido como un segundo hogar en red para los jóvenes en su conjunto, quienes "bajo un cierto orden y en un mismo lugar, concentran vínculos, información y entretenimiento. "Experiencias en última instancia" (Ciuffoli, C. y López, G.,2010:115). Como hemos visto, el motivo principal de uso es comunicarse, relacionarse e interactuar, pero el entretenimiento y la diversión son los ejes centrales en sus prácticas de ocio digital a través de estas redes. La falta de formación en relación a los usos realmente beneficiosos de estos medios sociales, en términos de aprendizaje, participación, enriquecimiento colectivo, colaboración en red y desarrollo, deja patente que el uso que los jóvenes hacen de las TIC, Internet y las RSV permanezca en un plano superficial.

Es tal el éxito de estas RSV que según el estudio “Connected Technology Report 2012" elaborado por el Cisco, el 90\% de los jóvenes entre 18 y 29 años, al despertarse, lo primero que hace es revisar su teléfono móvil, actualizar el correo electrónico, enviar mensajes y entrar en sus perfiles en redes sociales. Incluso dos de cada cinco jóvenes afirman que "se sentirían ansiosos, como si les faltase una parte de ellos si no pudieran usar su smartphone para estar conectados”. De ahí que, nos encontramos ante 
un fenómeno de ocio muy candente que al tratarse de un ocio digital social, móvil, lúdico y transmedia debe de ser revisado constantemente. Aún se desconocen sus verdaderas influencias (positivas y negativas) y son muchas las cuestiones que quedan por resolver.

\subsection{Ocio Digital Lúdico: videojuegos}

La importancia de la dimensión lúdica en la percepción social de lo que significa el ocio para las diferentes sociedades siempre ha sido mayúscula. Y esta importancia es mayor aún cuando hablamos de algunas etapas concretas de la vida, en el que el valor del juego tiene una gran importancia como son la infancia y la juventud.

En el ámbito del ocio digital, lo lúdico está directamente vinculado con los videojuegos, sector que ha ido en crecimiento desde su aparición en 1973. Desde entonces, han tenido un crecimiento constante y se han convertido en un fenómeno de masas que forma parte de la vida cotidiana y se presenta como la forma más común de experimentar el ocio digital. A medida que los avances tecnológicos han ido ofreciendo mejoras en la calidad de imagen, mayor realismo, así como nuevos dispositivos y programas, formas de acceso, interconectividad entre jugadores, intercambio de contenidos y juegos masivos en red, este tipo de ocio se ha consolidado para diferentes públicos (juegos infantiles, adolescentes, jóvenes, adultos, personas mayores) como una alternativa muy sugerente de ocio y en ciertos de estos colectivos el camino de entrada al mundo digital.

A lo largo de 2012, más de un tercio (35\%) de los españoles entre 16 y 64 años compró algún videojuego (ISFE, 2012), lo que sitúa a España como el cuarto país europeo en cuanto a consumo (ADESE, 2013), dándose la mayor penetración del videojuego en el tramo de edad de 7 a 34 años (ADESE, 2013). Asimismo, se ha podido constatar que esta creciente penetración no es causa de aislamiento o abandono de otras actividades (UOC, 2009). La mayoría $(89,2 \%)$ reconoce que prefiere salir con los amigos a jugar a videojuegos, lo que nos indica que los videojuegos no son utilizados o no se convierten necesariamente en sustitutos de la vida social cotidiana de los adolescentes. Entre los jugadores habituales el 47,1\% deja de jugar con videojuegos por llevar a cabo otras actividades como salir con amigos (44,3\%) y estudiar/trabajar $(25,7 \%)$ (ADESE, 2009).

Se detectan diferencias significativas en relación al género, con 59\% de hombres entre los jugadores españoles (ADESE, 2012). Este dato se confirma en otros estudios (González-Anleo. et al, 2011), que reafirman que los juegos de ordenador y juegos electrónicos en general son una actividad más masculina que femenina. Destacan también las diferencias de género existentes en relación a la frecuencia de juego, siendo las mujeres jugadoras más ocasionales que los varones (ADESE, 2011).

En relación a las motivaciones, el estudio de GFK destaca como principal motivación del juego, la diversión y el entretenimiento. A ésta, se añaden otras motivaciones en función de las tipologías de jugadores como auto superación, sensación de control, competitividad, vivir en mundos de fantasía (jugadores intensivos), concepto social, el valor funcional del juego como ejercitarse o hacer deporte mientras que la competición está en un segundo plano (jugadores casuales). La presencia de los juegos en redes sociales tales como Facebook plasma el amplio crecimiento e importancia de los juegos sociales, desde el ya famoso Farmville (que a inicio de 2010 tenía 83.099.000 usuarios mensuales activos), al actual Candy Crush con diez millones de usuarios jugando de manera diaria. Es decir, la potencia de los denominados juegos o videojuegos sociales es enorme.

La relación entre los videojuegos y los soportes sobre los que se juega ha sido fundamental desde los inicios de su historia. Desde la televisión, a los ordenadores, pasando por las consolas portátiles cada incorporación de nuevos soporte ha suscitado no solamente cambios en los propios juegos y su naturaleza, sino en los públicos a los que estos se dirigen. El fenómeno de la movilidad no se ha quedado atrás. Según datos de newzoo a finales de 2012, la plataforma en la que más se juego son las consolas, pero en referencia al tiempo de juego, más del $60 \%$ del mismo se hace en juegos móviles o sociales. Destacan también que en lo referido a España los jugadores son fundamentalmente de smartphone y consolas portátiles y menos de tablet. 


\subsection{Ocio Digital Móvil: conectividad sin límites a través de los smartphones}

El fenómeno móvil, actualmente bajo la dominación del smartphone o teléfono inteligente, parte de la idea de "la existencia de una cultura juvenil que halla en la comunicación móvil una forma adecuada de expresión y reafirmación" (Castells et al., 2007:205). Prácticamente desde el comienzo del desarrollo de la telefonía móvil se comenzó a hablar de "ocio móvil" que incluía desde los servicios de futurología o sexo telefónico, pasando por productos que pudieran considerarse de ocio y que funcionaran a través de los mismos (juegos, chats, etc.) (Castells et al., 2007:177-180), hasta los smartphone de hoy en día que incluyen prácticamente todas las actividades que hace unos años se desarrollaban en los ordenadores de mesa. Es decir, el teléfono móvil ha pasado de concebirse como una herramienta cuya principal función es comunicarse y hablar, a instaurarse como un dispositivo que ha evolucionado hasta estar más cerca de un ordenador personal portátil (Consejo Nacional Consultivo de CyberSeguridad, 2012). Por otro lado, para 3 de cada 4 jóvenes la comunicación es el motivo más importante para conectarse a Internet (Fundación Telefónica, 2012) por lo que, de lo que no cabe duda es de que los nuevos dispositivos móviles están modificando los hábitos de consumo de contenidos siendo ahora: personalizados, en movilidad, deslocalizados, ubicuos, de uso informal y casual, intensivo y social (Fundación Telefónica, 2011).

Es interesante destacar que, a esos jóvenes adolescentes que a comienzos del nuevo siglo eran usuarios intensivos y los primeros en adoptar un modo de vida móvil, se les atribuye "la invención" de usos no previstos por los diseñadores de dicha tecnología (Castells et al., 2007:208). Ya entonces, para los jóvenes europeos en general "lo más importante de la comunicación móvil es que permite la construcción y el mantenimiento de sus redes sociales" (Oskman y Rautiainen, 2002 y Valor y Sieber, 2004) para el caso de España citados en Castells et al., 2007).

En Europa, la penetración de los teléfonos móviles pasó de un $1 \%$ entre $1992-1993$ a un $71,55 \%$ en 2004, siendo además los jóvenes europeos de entre
15 y 25 años los que alcanzaban porcentajes más elevados de hasta el 77,2 \% (Castells et al., 2007: 207). La edad de su primer teléfono móvil se incrementa significativamente a partir de los 10 años hasta alcanzar el 92,1\% en la población de 15 años (INE, 2010). Lo que significa que, a día de hoy, los jóvenes españoles son usuarios de móviles más que experimentados. Actualmente España es líder de la UE en penetración de smartphones y los últimos informes arrojan datos como que el 68,3\% de los internautas de entre 16 y 24 años tiene un teléfono inteligente, el 25,4\% está 24 horas conectado y 1 de cada 5 jóvenes se conecta una vez acostados (Fundación Telefónica, 2012).

Así, hoy en día, el smartphone permite usos avanzados como gestionar el correo electrónico o acceder a las redes sociales, basados principalmente en una navegación adaptada por Internet, para lo cual disponen de pantallas amplias y táctiles, teclados completos (querty) y con una velocidad de navegación de grandes velocidades (3G o superior) (INTECO, 2011). Por lo tanto, hay que señalar que su penetración y uso por parte de los jóvenes especialmente para el ocio, ha estado muy vinculado a la accesibilidad económica, es decir, al acceso a tarifas asequibles para la mayoría de la población a la que ahora se considera la "combinación ganadora" (Fundación Telefónica, 2012), la convergencia de Internet y la telefonía móvil a través de los smartphone. A través de estos dispositivos, los jóvenes han "saltado" una de las barreras identificadas al disfrute del ocio digital como era el acceso a la tecnología y posteriormente al tipo de ocio a las que te da acceso y sus diferentes usos, ya que en función de los usos, nos encontramos con diferencias sociales en la forma de percibir el ocio (Castells et al., 2007).

Teniendo en cuenta a los menores (hasta 16 años) como los futuros jóvenes, cabe destacar que los usuarios intensivos de aplicaciones de smartphone relacionan su uso con lo que ellos identifican con actividades de ocio, incluyendo entretenimiento (69 $\%)$, uso de redes sociales (64\%), comunicarse $(54,5$ $\%)$ y uso en tiempo libre (49,3\%) (INTECO, 2011). Al igual que anteriormente, hay que señalar la dificultad de dar respuesta a algunas de las preguntas planteadas en este estudio ya que las 
clasificaciones de las actividades siguen centrándose en un ocio entendido como actividad, tiempo libre u ociosidad, y no como experiencia y que, además, son diferentes de unos estudios a otros.

Por lo tanto, el acceso va a ser determinante. Hoy en día el 66,7\% de los internautas entre 16 y 24 años utiliza su teléfono móvil para acceder a Internet, cuando lo hace desde fuera de su vivienda habitual o centro de trabajo (INE, 2012 en Fundación
Telefónica, 2012). De hecho, el 2012 se considera como el año en que realmente se afianza la Banda Ancha Móvil (BAM) creciendo por encima de un $150 \%$. Este crecimiento se atribuye precisamente a la "llegada" de franja de los jóvenes, ya que 2 de cada 3 jóvenes de entre 16 y 24 años poseen un smartphone con conexión, lo que ha supuesto un crecimiento de más de un $200 \%$.

\section{Conclusiones}

La cultura digital que nos rodea ha reinventado la vida en general y la esfera del ocio en particular, y los jóvenes nacidos en la era digital y que centran el interés de esta reflexión ya han sido bautizados como Generación@ (Feixa,2002), Nativos Digitales (Prensky, 2001), Generación Nintendo (Howard, 2003), Generación Net (Tapscoot, 1998), Generación Interactiva (Bringué y Sádaba, 2009), Generación A (Coupland, 2010) etc.

Independientemente de la originalidad o acierto de cada uno de los conceptos propuestos, todos ellos hacen referencia a un grupo de personas nacidas en torno a la década de los 90, rodeados de tecnologías digitales, con formas de relación similares y preocupaciones, intereses $\mathrm{u}$ opiniones en muchas ocasiones comunes. Se refieren a jóvenes que están creciendo en un contexto social, cultural y educativo distinto, especialmente por la presencia continua y constante de la tecnología, y que presentan rasgos distintivos respecto a otras generaciones. Características tecnológicas que afectan a todos los ámbitos de su vida y especialmente al referido al ocio y que en esta edad es de una importancia vital. El contexto digital en el que estos jóvenes se han desarrollado y madurado supone un uso simultáneo de diferentes herramientas tecnológicas, pantallas y códigos que cobra especial importancia en sus dos ámbitos fundamentales: la escuela y el ocio.

En esta ocasión el ocio es el aspecto humano que nos ocupa analizar y se observa como la irrupción de las TIC ha supuesto un cambio en la tradicional relación entre los públicos y la industria del ocio, transfigurando el comportamiento de las audiencias $\mathrm{y}$, por consiguiente, las formas de consumo y prosumo de contenidos digitales de ocio fundamentales para la cultura juvenil como son la música, la televisión, los videojuegos, los comics, o los libros. La transformación y la posibilidad multitarea de los dispositivos tecnológicos más comunes (ordenadores, móviles, tabletas...) ha hecho más patente aún la dispersión de las barreras entre los tiempos, espacios y usos del ocio. Esto permite a los jóvenes simultanear en tiempo real espacios de ocio con espacios de estudio e incluso incorporar tiempos (viajes y tiempos de espera) a la esfera del tiempo libre y del ocio modificando conductas muy asentadas en generaciones anteriores.

Incluso las actividades tecnológicas tradicionales de ocio del siglo XX, como ver la televisión, se han visto modificadas por este fenómeno ya que durante el tiempo de visionado de un contenido en la TV se produce una elevada tasa de uso tanto de los tablet como de los smartphone en lo que podríamos llamar la televisión social (Nielsen, 2011 citado en Fundación Telefónica, 2011). Este hecho provoca una superación de las diferencias entre los diversos soportes y contenidos desde el punto de vista de la industria. El usuario lo que quiere es consumir, comentar, prosumir y crear nuevos contenidos digitales a partir los contenidos ofrecidos "tradicionalmente", cuando, donde y sobre el soporte que quieren.

Por consiguiente, en la actualidad, es tal la variedad de dispositivos y pantallas existente, que el hábito de permanecer a la espera delante del televisor para ver un programa, a la misma hora está desapareciendo, y en plena transición hacia una cultura digital la frase "nos vemos la próxima semana, en el mismo canal y a la misma hora" ha dejado de tener sentido para las 
nuevas generaciones de jóvenes multitarea, sociales, móviles y conectados.

Lo realmente importante es que los consumidores cobran especial protagonismo como productores de contenidos. Algunos consumidores se tornan prosumidores (productores + consumidores), se apropian de sus personajes favoritos y expanden aún más sus mundos narrativos. Las posibilidades que ofrecen los diferentes dispositivos han hecho que los usuarios cooperen activamente en este proceso. Los prosumidores del siglo XXI son activos militantes de las historias que les apasionan y cada día es más común observar este tipo de comportamientos. Lo que nos deja vislumbrar un cambio no sólo en el tipo de ocio juvenil, sino también en la manera de experimentar el ocio propio de una era digital.

La generación digital de jóvenes es una generación multitask (multitarea), que está acostumbrada a recibir múltiples inputs de información de forma rápida, simultanea, constante (sociedad infoxificada) y a través de muchos canales distintos a la vez. Son jóvenes que tienen la atención más diversificada (generación del click) y están acostumbrados a organizar la información de forma no lineal a partir de hipertextos. En este sentido, les gusta la acción constante y la inmediatez de recompensas como fruto de sus acciones, por lo que en muchas ocasiones no realizan un análisis crítico de la información recibida. Jóvenes interactivos que gracias a la interactividad como rasgo propio de las red de redes se posicionan ante las TIC no sólo como meros consumidores pasivos, sino también producen contenidos y participan en la red a través de diversos medios sociales como los blogs, las wikis, los foros de discusión o las redes sociales virtuales. Mantienen así activamente espacios digitales de comunicación, auto-presentación y de creación y se convierten en verdaderos prosumidores.

Tienen la necesidad de estar permanentemente conectados y comunicados en la distancia o incluso en la cercanía, a través del uso del móvil, la mensajería instantánea (chats o messenger) o de las redes sociales virtuales. La dimensión lúdica y la social son una parte crucial en el proceso de construcción de su identidad personal, y esta identidad digital (la elección de la foto del perfil por ejemplo) es simplemente una prolongación de sus vidas offline, las barreras entre lo virtual y lo real son difusas y por ello le dan tanto valor a la construcción de su imagen virtual como a la imagen real. En consecuencia, con la aparición de estos nuevos formatos de comunicación y socialización, sus prácticas discursivas comunicativas, la forma de escritura que emplean y, en definitiva, el lenguaje que usan ha cambiado por completo, originándose un lenguaje propio de los contextos de comunicación digital y descrito como "texto escrito oralizado", "habla escrita" o "ciberhabla". Son una generación que tiende hacia el uso de un nuevo género capaz de integrar lo oral, lo escrito y lo gestual (emoticonos). En la misma línea, desde el mundo del marketing digital se puede trasladar igualmente el concepto de SoLoMo (CuttS, 2011), para definir a jóvenes usuarios sociales (so), locales (lo) y móviles (mo), y practican un ocio SoLoMo.

Estos comportamientos de ocio provocados por el uso de la tecnología e Internet han posibilitado nuevas formas de disfrute donde la conectividad influye en las experiencias. Fuera y dentro de la misma pantalla muchos consumidores, aún una minoría, terminan en Internet horizontalizando el modelo de comunicación. Los adolescentes y jóvenes adultos son los mayores exponentes de la denominada "Generación C", que se resiste a consumir contenidos simbólicos en las mismas condiciones temporales y programáticas que las generaciones anteriores. $\mathrm{La}$ "C" encierra una serie de significados, como conectividad constante, colaboración, cocreatividad y curiosidad. "La fórmula "consumidor conectado colectivamente" puede sintetizar estos comportamientos que representan para algunos una nueva civilización bárbara en búsqueda de estatuto" (Igarza, 2013:151). García Fernández (2009) ofrece cinco características que determinan cómo son los jóvenes a los que nos referimos: multitarea, acción constante, fluidez en el manejo de la tecnología, prosumidores y conectados.

Por último, y en base a las ideas de Don Tapscott (1998) podemos afirmar que la Generación Net es cada vez más independiente y autónoma, tiene un papel activo como buscadores de información y se expresa libremente, exponiéndose a toda un amplia gama de ideas, opiniones y argumentos. Los jóvenes 
del siglo XXI tienen una representación positiva de la tecnología, y para ellos los medios sociales y en concreto las redes sociales virtuales son herramientas de comunicación cómodas, sencillas, "gratuitas" y espacios de "libertad e intimidad" donde expresarse libremente, alejados del control de los educadores y la familia. En resumen, podemos decir que la actual generación de jóvenes, es claramente diferente a las anteriores básicamente porque su desarrollo personal ha estado vinculado y favorecido (o no) en la denominada era digital, formando parte de la sociedad de la información y del conocimiento, lo cual no garantiza adultos más informados ni con mayores conocimientos.

Por lo tanto y como era de esperar, los jóvenes de hoy tienen una gran fluidez en el manejo de la tecnología, lo cual no determina que realicen un uso adecuado de ella, y su principal motivación de uso es el ocio, entendido este como entretenimiento y pasatiempo. Así pues, el uso generalizado de las TIC es más bien pasivo y superficial. Pese a que la red posibilite el poder actuar como auténticos "prosumidores", la realidad plasma una generación de jóvenes que valoran muy positivamente el estar en permanente conexión, esto es, son jóvenes conectados, pero que desaprovechan y desconocen el potencial de su ocio en red. Son una generación que además de ser jóvenes interactivos, expanden su ser a través de múltiples medios y plataformas y ello queda patente en sus estilos de ocio.

En definitiva, esta reflexión lejos de dar respuestas definitivas, nos lleva a plantearnos nuevas preguntas. $\mathrm{Si}$ como se ha visto, por un lado se habla de "nuevos ocios", "ocio en red", "móvil" o "fast ocio"; "textos escritos oralizados", "narrativas transmedia" o "multitarea"; espacios "virtuales" y "reales", online y offlline; y de una generación SoLoMo, "prosumidora" y con todo tipo de denominaciones: @, net, A, Nintendo o Millenials, ¿podemos hablar de un "ocio digital”? Las comillas son delatoras. Definitivamente, los límites de algunos conceptos clásicos se diluyen de tal manera y la tecnología está embebida de tal forma en la vida diaria de toda una generación (y las siguientes) que demanda hacer toda una reformulación de conceptos, entre ellos el ocio y las formas de experimentarlo. Sin duda parece evidente que no podemos seguir preguntando: ¿utilizas Internet para actividades de ocio? o ¿cuántas horas diarias lo utilizas?

\section{Referencias}

- Abad Galzacorta, M. (2009). ACCEPTuR, un modelo de ACeptación teCnológica para ExPeriencias TuRísticas. (Tesis doctoral sin publicar). Donostia-San Sebastián. Universidad de Deusto.

- ADESE (2011). El videojugador español: perfil, hábitos e inquietudes de nuestros gamers. Disponible

en:

http://www.slideshare.net/AleixRisco/2011 -el-videojugador-espaol-perfil-hbitos-einquietudes-de-nuestros-gamers

- ADESE (2012). Anuario de la industria del videojuego. Disponible en: http://www.aevi.org.es/anuario2012/

- Aristegui, I. y Ayerbe, M. (2010) El valor del trabajo. En Elzo, J. y Silvestre, M. (Dirs.). Un individualismo placentero y protegido. Cuarta Encuesta Europea de Valores en su aplicación a España (pp. 104-105). Bilbao: Universidad de Deusto.
- Bauman, Z. (1999). Modernidad líquida. Buenos Aires: Fondo de Cultura Económica.

- Bryce, Jo (2001). The technological Transformation of Leisure. Social Science Computer Review, 19, 7-19.

- Bucy, E.P. (2004). Interactivity in society: Locating an elusive concept. The Information Society, 20, 373-383.

- Castells, M. (1997). La era de la información. Economía, cultura y sociedad. La sociedad red. Madrid: Alianza.

- Castells, M. (2001). La Galaxia Internet. Plaza \& Janés.

- Castells, M., Fernández-Ardèvol, M,. Linchuan Qiu, J. y Sey, A. (2007). Comunicación móvil y sociedad, una perspectiva global (pp. 205206). Barcelona: Ariel y Fundación Telefónica. 
- CISCO (2012). Connected Technology Report 2012.

- Ciuffoli, C. y López, G. (2010). Facebook como paradigma de la alfabetización digital en tiempos de la barbarie cultural. En A. Piscitelli, I. Adaime y I. Binder, I. (Eds.) El proyecto Facebook y la Posuniversidad. Sistemas operativos sociales y entornos abiertos de aprendizaje ( $1^{\mathrm{a}}$. ed. pp. 111-128). Madrid: Ariel y Fundación Telefónica.

- Consejo Nacional Consultivo de CyberSeguridad (2012). Informe Malware en smartphones. Informe disponible en: http://www.s21sec.com/descargas/Malware _Smartphones_CNCCS\%20.pdf.

- Cornella (2009). Infoxificación: Buscando un orden en la información. Barcelona: Infonomia.

- Cuenca Cabeza, M. (2000). Ocio Humanista. Dimensiones y manifestaciones actuales del ocio. Documentos de Estudios de Ocio, 16. Bilbao: Universidad de Deusto.

- Cuenca Cabeza, M. (2004): Pedagogía del ocio: modelos y propuestas. Bilbao: Universidad de Deusto

- Cuenca Cabeza, M. (2006): Aproximación multidisciplinar a los Estudios de Ocio. Documentos de Estudios de Ocio, 31. Bilbao: Universidad de Deusto.

- Cuenca Cabeza, M.; Aguilar Gutiérrez, E.; Ortega Nuere, C. (2010): Ocio para innovar. Documentos de Estudios de Ocio, 42. Bilbao: Universidad de Deusto.

- Elogia Marketing e-Commerce y Iab Spain Research (2013): IV Estudio anual Redes Sociales.

- Fundación Telefónica (2012). SIE 2012. La Sociedad de la Información en España. Madrid.

- García Álvarez, E.; López Sintas, J. y Samper Martínez, A. (2012): "Retos y tendencias del Ocio Digital: transformación de dimensiones, experiencias y modelos empresariales”. ARBOR Ciencia, Pensamiento y Cultura, vol.188-754, marzo-abril), pp 395-407. Disponible en: http: / / arbor.revistas.csic.es/index.php/arbor /article/viewArticle/1471 (Fecha de consulta: 15/03/2013).
- García Fernández, F. (2009). Nativos Interactivos. Los adolescentes y sus pantallas: reflexiones educativas. Foro Generaciones Interactivas 2009. Disponible

en:

www.generacionesinteractivas.org (Última consulta 15/02/2012).

- González-Anleo, J.M. (2011) Jóvenes españoles 2010. Madrid: Fundación SM.

- GFK Emer Ad Hoc Research (mayo, 2010). ¿Cómo se proyecta el videojuego del futuro? Disponible en http: / / www.adese.es/pdf/videojuegosimport .pdf

- Igarza, R. (2009). Burbujas de ocio. Nuevas formas de consumo cultural. Buenos Aires: La Crujía.

- INTECO y ORANGE (2011) Estudio sobre hábitos seguros en el uso de smartphones por los niños y adolescentes españoles. Disponible en: http://www.inteco.es/

- ISFE, 2012. VIDEOGAMES IN EUROPE: CONSUMER STUDY. European Summary Report. Disponible en http://www.isfe.eu/sites/isfe.eu/files/attach ments/euro_summary__isfe_consumer_study.pdf

- Henry JENKINS (6 nov 20012). Why the Matrix matters. (Blog) Disponible en: http: / / www.technologyreview.com/view/4 02277/why-the-matrix-matters/ (Fecha de consulta: 02/02/2014)

- Madariaga Ortuzar, Aurora y Cuenca Amigo, Jaime (2011): Los valores del ocio: cambio, choque e innovación. Documentos de Estudios de Ocio, 43 Bilbao: Universidad de Deusto.

- Moreno A. y Rodríguez, E. (2011). Cifras Jóvenes. Sondeo de opinión. Madrid: Injuve. Accesible

en

http://www.injuve.es/sites/default/files/20 $12 / 27 /$ publicaciones/Sondeo $\% 202011$ 1b.pdf

- Nimrod, G. y Adoni, H. (2012). Conceptualizing E-Leisure . Loisir et Société / Society and Leisure, 35 (1), pp. $31-56$. 
- Observatorio de la Juventud en España (2010). Juventud en Cifras. Ocio y Tiempo Libre. Madrid: Ministerio de Sanidad, Política Social e Igualdad.

- Observatorio Juventud de España y Centro de Investigaciones Sociológicas (2011): Cifras y jóvenes. Sondeo de opinión. Jóvenes y Tic. Madrid: INJUVE.

- Observatorio de la Juventud (2012).Informe Juventud en España. Madrid: INJUVE Disponible

http: / / www.injuve.es/sites/default/files / 20 13/26/publicaciones/IJE2012_0.pdf

- ONTSI (Observatorio Nacional de las Telecomunicaciones y la Sociedad de la información (2012): Los contenidos digitales en España. Informe Anual 2011. Disponible en:http://www.ontsi.red.es/ontsi/sites/defa ult/files/informe contenidos digitales edicio $\underline{\text { n2012.pdf }}$

- Pine, J. B. y Gilmore, J. H (2000): La economía de la experiencia , Barcelona: Granica.

- Prensky, M. (2001): "Digital Natives, Digital Inmigrants. On the Horizon, MCB University Press, 5.
- Rojek, C. y Urry. (2000). Touring Cultures. Transformations of Travel and Theory. London and NY: Routledge.

- San Salvador del Valle Doistua, R. (2009): "El tiempo acelerado". El País. Disponible en: http: / / elpais.com/diario/2009/11/01/paisv asco/1257107999 850215.html (Última consulta: $02 / 03 / 2013)$.

- Sibilia, P. (2008). La Intimidad como Espectáculo. Buenos Aires: Fondo de Cultura Económica.

- Turkle, S. (2012): ¿Conectados pero solos?. TED. Disponible en: http://www.ted.com/talks/sherry turkle al one together.html (Fecha de consulta: 26/02/2013).

- Van Dijk, J. (1999): The network society. Social aspects of new media. London: Sage.

- World Leisure Recreation Association (1994): Carta para la Educación del Ocio. En S. Gorbeña; V.J. González. y Y. Lázaro. (1997). El Derecho al ocio de las personas con discapacidad (pp. 243-252). Documentos de Estudios de Ocio, 4. Bilbao: Universidad de Deusto.

\begin{abstract}
Apoyos
Este artículo se enmarca en el subproyecto nacional I+D+i (EDU2012-39080-C07-03)de carácter coordinado titulado: "De los tiempos educativos a los tiempos sociales: participación de los jóvenes en la creación y gobernanza de sus espacios de ocio como factor de desarrollo personal" que está siendo elaborado por el centro investigación muoltidisciplinar, el Instituto de Estudios de Ocio de la Universidad de Deusto.
\end{abstract}

\title{
Forma de Citación
}

VIÑALS BLANCO, Ana; ABAD GALZACORTA, Marina y AGUILAR GUTIÉRREZ, Eduardo: Jóvenes conectados: Una aproximación al ocio digital de los jóvenes españoles. Revista Communication Papers, $\mathrm{N}^{\circ} 4$, páginas 52 a 68. Departamento de Filología y Comunicación de la Universidad de Girona. Recuperado el _ de_ de $2 \ldots$ de: http://www.communicationpapers.es 